2020-04

\title{
Geometrical investigation of a U-shaped oscillating water column wave energy device
}

\author{
Ning, D-Z
}

http://hdl.handle.net/10026.1/15596

10.1016/j.apor.2020.102105

Applied Ocean Research

Elsevier BV

All content in PEARL is protected by copyright law. Author manuscripts are made available in accordance with publisher policies. Please cite only the published version using the details provided on the item record or document. In the absence of an open licence (e.g. Creative Commons), permissions for further reuse of content should be sought from the publisher or author. 
1 Geometrical Investigation of a U-shaped Oscillating Water

4 a State Key Laboratory of Coastal and Offshore Engineering, Dalian University of Technology, Dalian, 116024, China

${ }^{\mathrm{b}}$ School of Engineering, Computing and Mathematics, University of Plymouth, Drake Circus, PL48AA, Plymouth, UK

Abstract: Hydrodynamic performance of a fixed U-shaped Oscillating Water Column (U-OWC) Wave Energy Converter is numerically investigated. Based on the time11 domain higher-order boundary element method (HOBEM), a two-dimensional fully nonlinear numerical model is implemented to simulate the nonlinear wave interaction with a U-OWC device. In the model, the inner-domain-source method is adopted to

14 generate the incident waves and a linear pneumatic model is used to determine the air 15 pressure which is imposed on the free surface inside the chamber. The numerical model is well validated against the published experimental data of the free surface elevation

17 at the chamber center, air pressure inside the chamber and hydrodynamic efficiency.

18 Further, the present model is applied to study the effects of geometrical parameters 19 (including the vertical duct height, vertical duct width and wall thickness) on the hydrodynamic performances of the U-OWC device. The results indicate that

21 geometrical parameters of the vertical duct have significant influence on the air pressure 22 inside the chamber and the hydrodynamic efficiency. The hydrodynamic efficiency and 23 air pressure inside the chamber are found to increase with the increase of both vertical 24 duct height and thickness of wall I. However, the increasing rate of the efficiency slows 25 down gradually with the thickness of wall I increasing, which indicates that a 26 comprehensive consideration of the construction cost and hydrodynamic performance

\footnotetext{
* Corresponding author. E-mail: rqwang@dlut.edu.cn
} 
1 is needed in the design and construction of a U-OWC device.

2 Keywords: U-OWC; Nonlinearity; HOBEM; Hydrodynamic efficiency; Wave energy

\section{1. Introduction}

Energy supply and climate change have plagued human society for decades. Hence, it is significant for human being to exploit the clean renewable energies. In this regard, ocean energies have attracted a large amount of attention because of their viable ocean energies due to its high energy flux density and constructive hydrodynamic interaction of multi-converters.

A great deal of research on wave energy converters (WECs) has been carried out.

11 Therein, the Oscillating Water Column (OWC) WEC appears to be one of the most successful and the ripest types. The OWC-WECs were investigated theoretically, experimentally and numerically by Evans and Porter [2], Martins-rivas and Mei [3],

14 Zheng et al. [4] Deng et al. [5], Ning et al. [6], Wang and Ning [7], and so on. In recent 15 years, the floating OWC-WECs were proposed to expand the application area of the 16 OWC devices, such as Falcão et al. [8], Gomes et al. [9], Crespo et al. [10], He and 17 Huang [11]. Furthermore, The OWC-WECs with dual chambers were investigated by 18 Rezanejad et al. [12], He et al. [13], Ning et al. [ 14, 15], etc., which can broaden the 19 effective frequency bandwidth by inducing more resonant frequencies of the device. 20 Not only various types are proposed, but also the geometrical design details are 21 investigated to further improve the performance and survivability of OWC devices. For 22 instance, the effects of front wall draft, slope degree of front wall and chamber width 23 on the hydrodynamic performance of the OWC devices were investigated in [16-18]. 24 Based on those crucial researches, prototypes of the OWC-WECs have also been built 25 in several countries [19-21].

26 A variant OWC device was proposed by Boccotti [22], named as U-shaped 27 oscillating water column (U-OWC) device, consisting of an air chamber, a Power Take- 
1 Off (PTO) system, which often simulated by an orifice located at the air chamber celling, 2 and an additional vertical duct as shown in Fig. 1. The vertical duct changes 3 significantly the excitation form of the U-OWC device with respect to the conventional

4 OWC device. The incident wave trains cannot propagate into the inner chamber, but the 5 hydrodynamic force exerted at the upper opening of the vertical duct induces an up and 6 down motion of the water column and then a reciprocating air-flow through the orifice. 7 Compared to the conventional OWC device, the eigenperiod of the U-OWC device and 8 the air pressure inside the U-OWC chamber are larger [22]. Additionally, the U-OWC 9 device can avoid sand entering into the chamber and can also decrease air inhalation at 10 bottom opening, which benefits the security of the device.

12

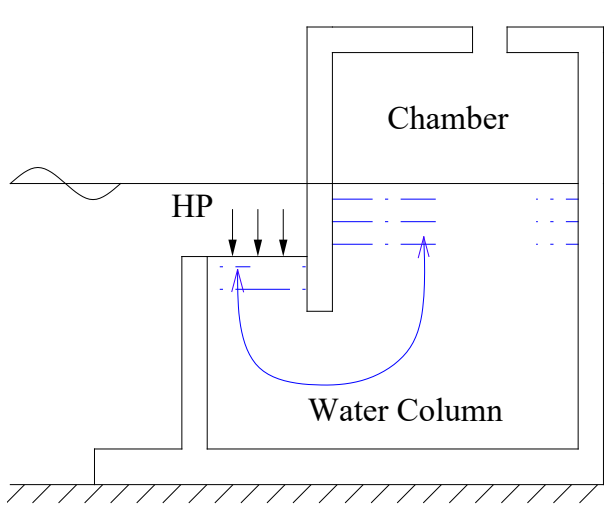

Figure 1 A schematic of the U-OWC device.

Hydrodynamic investigations on the U-OWC device have been carried out theoretically, numerically and experimentally since its concept was put forward. Initially, Boccotti [23] proposed the concept of the U-OWC device and pointed out its advantages. Successively, the related theoretical derivations and numerical simulations were performed. One theoretical framework of inner water column oscillation was introduced by Boccotti [24] according to the unsteady Bernoulli theorem. Later, Boccotti et al. [25] performed a laboratory experiment to validate the reliability of the theoretical model. Malara and Arena [26] developed a numerical model by coupling the eigenfunction expansion method outside the U-OWC device based on linear wave 
1 theory with the unsteady Bernoulli equation in the internal region. Malara et al. [27]

2 revised the underrated head losses in the previous theoretical model. They introduced

3 the unsteady head losses into motion equation based on the acceleration-based

4 technique and implemented a field experiment to calibrate it. Soon afterwards, Malara

5 et al. [28] developed a three-dimensional (3D) numerical model of the U-OWC device

6 based on the boundary integral equations method in the frequency domain and linear

7 wave theory. Spanos et al. [29] employed a statistical linearization method to describe

8 the motion of the U-OWC. Malara and Arena [30] proposed a semi-analytical approach

9 to calculate the hydrodynamic efficiency of the U-OWC WEC array. They found that

10 the array reduces the hydrodynamic efficiency of the U-OWC device with respect to

11 the isolated U-OWC device. Strati et al. [31] adopted the Maximum Power Point

12 Tracking (MPPT) algorithm to control the coupling between the U-OWC device and

13 the PTO system. Some experiments were also conducted in addition to the above 14 numerical models. Vyzikas et al. [32] experimentally studied the hydrodynamic 15 performance of four variants of a three-chamber OWC model with and without the PTO 16 system. The experimental data indicated that the hydrodynamic efficiency of the U17 OWC device is superior to the traditional OWC device, especially for the U-OWC device with a bottom slope. Arena et al. [33] conducted a field experiment to analyze

19 the interaction between the Dielectric Elastomer Generator (DEG) PTO system and the 20 U-OWC device. From the engineering perspective, Boccotti [34] explored some 21 regulations to guide the overall design of the full-scale U-OWC WEC. Arena et al. [35] 22 discussed the crucial issues on the design and construction stages of the U-OWC device. 23 Monte Carlo simulation was utilized to determine the inner free surface displacement 24 and the maximum load exerting on the walls, and further to evaluate the working 25 condition of the PTO system and the safety of the air chamber.

26 Most of the previous studies were mainly focused on the theoretical models and 27 investigating the effects of the PTO system on the hydrodynamic efficiency. There is a 28 lack of investigation on the influence of the geometrical parameters on the 
1 hydrodynamic performance of the U-OWC device. Moreover, the theoretical studies of

2 the U-OWC device were mostly based on linear wave theory. However, the

3 hydrodynamics of the wave interaction with U-OWC device is quite complicated and

4 nonlinear. In the present study, based on the fully nonlinear potential flow theory and

5 higher-order boundary element method (HOBEM), the effects of the geometrical

6 parameters (i.e., the vertical duct height, vertical duct width and barrier wall thickness)

7 on the hydrodynamic performance of a U-OWC device are numerically investigated.

8 The rest of the present paper is organized as follows. The numerical model is

9 described in section 2. Reliability and accuracy of the model are verified in section 3.

10 Effects of geometrical parameters on the hydrodynamic performance of the U-OWC

11 device are investigated in section 4. Finally, conclusions are summarized in Section 5.

\section{2. Numerical model}

The two-dimensional (2-D) fully nonlinear numerical wave flume based on the time-domain HOBEM developed by Ning et al. [36] to investigate the hydrodynamic performance of an OWC device is extended here to simulate the wave interaction with the U-OWC device. In the numerical model, an inner-source technique is adopted to generate the incident wave so that the re-reflection on the input boundary of the numerical wave flume (NWF) can be avoided. Meanwhile, a pneumatic model is used to determine the air pressure which is imposed on the free surface inside the chamber.

A schematic diagram of the NWF with a U-OWC wave energy converter is shown

21 in Fig. 2. Herein, a Cartesian coordinate system is set up with its origin $(O)$ at the intersection point of inner-source surface and undisturbed free surface, $x$-axis positive in the direction of incident wave propagation, and $z$-axis positive upwards. As shown in Fig. 2, $h$ is the still water depth; $a$ is the orifice width; $b, c_{\mathrm{I}}, c_{\mathrm{II}}, e$ and $l$ are the air chamber width, thickness of barrier wall I, thickness of barrier wall II, vertical duct

26 width and base length (before the vertical duct), respectively; $d, w$ and $s$ are the draught

27 of barrier wall II, vertical duct height and base height, respectively. 


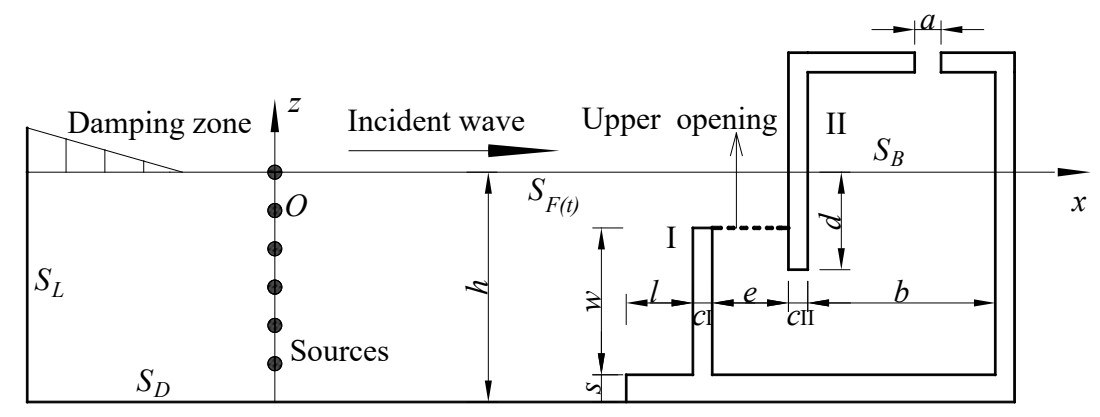

Figure 2 Schematic diagram of a U-OWC device in the numerical flume.

The assumptions of inviscid, incompressible and irrotational flow are usually used in the wave-OWC simulation and good accuracy is achieved [36-38]. Thus, the simulation of the present study about the wave- U-OWC interaction can also be described by potential theory, where velocity potential $\phi$ satisfies Laplace equation in the computational domain. Therein, due to the introduction of inner sources, Laplace equation was altered into Poisson equation as follows:

$$
\nabla^{2} \phi=q^{*}(z, t)=2 v \delta(x) \quad \text { in } \Omega
$$

in which $\nabla^{2}=\partial^{2} / \partial x^{2}+\partial^{2} / \partial z^{2}$ is the 2-D Laplacian operator, and $q^{*}(z, t)$ is the pulsating volume flux density of the internal sources (the horizontal position of vertical sources is located at $x=0), v$ the horizontal fluid velocity given by Stokes second-order analytical solution in the present study, which denotes the sources strength, $\delta(x)$ is the Dirac delta function, and $\Omega$ denotes the whole fluid domain, which is surrounded by the instantaneous free surface $S_{F(t)}$, the flume bottom $S_{D}$, the left boundary $S_{L}$ and the surface of the U-OWC device $S_{B}$ as shown in Fig. 2.

On the instantaneous free surface, an artificial damping coefficient is involved in the dynamic free surface boundary condition inside the chamber to consider the head losses due to the flow separation and vortex shedding caused by barrier wall I and II. The mixed Eulerian-Lagrangian method is employed to describe the instantaneous free surface with moving nodes in both $x$ and $z$ directions. A damping zone at the left boundary of the NWF is adopted to eliminate reflected waves from the U-OWC device. 
1 Thus, the kinetic and dynamic boundary condition can be rewritten as follows:

2

$$
\left\{\begin{array}{l}
\frac{\mathrm{d} \boldsymbol{X}(x, z)}{\mathrm{d} t}=\nabla \phi-\mu_{1}(x)\left(\boldsymbol{X}-\boldsymbol{X}_{0}\right) \\
\frac{\mathrm{d} \phi}{\mathrm{d} t}=-g \eta-\frac{P}{\rho}+\frac{1}{2}|\nabla \phi|^{2}-\mu_{1}(x) \phi-\mu_{2}(x) \frac{\partial \phi}{\partial n}
\end{array}\right.
$$

3 where $\mathrm{d} / \mathrm{d} t=\partial / \partial t+V \cdot \nabla$ is the material derivative, $V$ the velocity of water particle,

$4 X(x, z)$ the horizontal and vertical displacements of fluid particles on the free surface, $t$

5 the time, $\eta$ the free-surface elevation, $P$ the air pressure inside the chamber caused by

6 the oscillating motion of the water column, and $\mu_{1}(x)$ is the damping layer coefficient,

$7 \quad \boldsymbol{X}_{0}$ the initial position of fluid particles, $g$ the gravity acceleration, $\rho$ the fluid density.

$8 \mu_{2}(x)$ denotes the artificial damping coefficient. $\mu_{1}(x)$ and $\mu_{2}(x)$ can be defined as follows:

$$
\mu_{1}(x)=\left\{\begin{array}{cc}
\omega\left(\frac{x-x_{1}}{L}\right)^{2}, & x_{1}-L<x<x_{1} \\
0, & x \geq x_{1}
\end{array}\right.
$$

$$
\mu_{2}(x)=\left\{\begin{array}{cl}
\text { confirmation } & \text { inside of the chamber } \\
0 & \text { outside of the chamber }
\end{array}\right.
$$

11 where $\omega$ denotes the angular frequency of the incident wave, $L$ the length of the damping layer, $x_{1}$ the starting position of the damping zone, and 'confirmation' means that the specific value need to be determined by the trial-and-error method [6].

It is assumed that the flume bottom $S_{D}$, left boundary $S_{L}$ and body surface $S_{B}$ are the impermeable fixed boundary, and the fluid never separates from those boundaries. Thus, the water particle speed is equal to 0 in its normal direction.

$$
\frac{\partial \phi}{\partial \boldsymbol{n}}=0 \text { on } S_{D}, S_{L} \text { and } S_{B}
$$

where $\boldsymbol{n}$ is the outward normal unit vector on those solid boundaries.

The above-mentioned problem is not only a boundary value problem, but a timedependent problem. The initial condition should be given to solve aforementioned equations in time domain.

$$
\left\{\begin{array}{l}
\left.\phi\right|_{t=0}=0 \\
\left.\eta\right|_{t=0}=0
\end{array} \quad \text { on } \quad S_{F(t)}\right.
$$


There is a strong coupling between the air and water inside the U-OWC chamber

2 because the air pressure is directly related to the oscillation of the free-surface inside

3 the chamber. The numerical model assumes a linear relationship between the air

4 pressure and the air flow velocity through the duct. The air pressure inside the chamber

5 can be expressed as follows:

6

7

$$
P(t)=C_{\mathrm{dm}} U_{\mathrm{d}}(t)
$$

where $C_{\mathrm{dm}}$ denotes the linear pneumatic damping coefficient and $U_{\mathrm{d}}$ the air flow velocity at the orifice. Due to small-scale simulation considered, the air inside the chamber is assumed to be incompressible [14]. The detail information about the pneumatic model can be found in [36].

The above boundary value problem can be solved by HOBEM. Herein, to avoid the numerical instabilities, the free surface is re-meshed and updated at each time step and the quadratic shape function interpolation is adopted to compute the velocity potential and spatial position of new nodes. The more details about the HOBEM can be referred to [36].

The hydrodynamic efficiency is defined as a rate of the captured energy against the incident wave energy per unit width. The average captured energy by the U-OWC device during one period can be calculated by

$$
P_{\text {cap }}=\frac{1}{T} \int_{t}^{T+t} Q(t) P(t) d t=\frac{1}{T} \int_{t}^{T+t} b \overline{\dot{\eta}}(t) P(t) d t=\frac{1}{T} \int_{t}^{T+t} a U_{\mathrm{d}}(t) C_{\mathrm{dm}} U_{\mathrm{d}}(t) d t
$$

where $T$ is the wave period, $Q(t)=b \bar{\eta}(t)=a U_{\mathrm{d}}(t)$ is the airflow rate through the orifice and $\overline{\dot{\eta}}(t)$ is the time-dependent mean vertical velocity of the free surface inside the chamber.

Based on the incident wave conditions, the wave nonlinearity is weak. Thus, the incident average energy flux per unit width can be calculated based on the linear wave theory:

$$
P_{\text {inc }}=\frac{1}{2} \rho g A^{2} C_{g}
$$


1 where $A$ is the incident wave amplitude, $C_{g}=d \omega / d k$ the wave group velocity, $k$ the wave

2 number. The hydrodynamic efficiency can be calculated as:

$$
C_{\mathrm{w}}=P_{\text {cap }} / P_{\text {inc }}
$$

\section{3. Model validation}

In this section, the U-OWC numerical model is validated by comparing with the published experimental data [32]. Fig. 3 depicts the experimental setup of the U-OWC

10 those in the experiment. It's noteworthy that the orifice width in the numerical model

11 is determined by assuming that the opening ratio in the numerical model is equal to that

12 in the experiment. This paper mainly concerns the hydrodynamic performance of the

13 U-OWC device subjected to regular incident waves. The incident wave frequencies are

$140.57,0.51,0.465$ and $0.385 \mathrm{~Hz}$ corresponding to wave heights of $0.122,0.096,0.088$

15 and $0.159 \mathrm{~m}$, respectively. The damping coefficient $\mu_{2}=0$ and the pneumatic damping 16 coefficient $C_{\mathrm{dm}}=19 \mathrm{~kg} /\left(\mathrm{m}^{2} \cdot \mathrm{s}\right)$ are adopted by the trial and error method [6]. The left 17 damping layer is set as 1.5 times wavelength $\lambda$ (total length of NWF is 5 times 18 wavelength). The simulation is based on potential theory, so generic personal computer 19 is enough to complete the calculation, where an i7 CPU with six cores is used.

Table 1 Geometrical parameters of U-OWC device (m).

\begin{tabular}{llllll}
\hline$a$ & $b$ & $c_{\mathrm{I}}$ & $c_{\text {II }}$ & $d$ \\
\hline
\end{tabular}




\begin{tabular}{ccccc}
\hline $9.6 \times 10^{-4}$ & 0.286 & 0.024 & 0.024 & 0.482 \\
\hline$e$ & $l$ & $w$ & $s$ & $h$ \\
0.143 & 0.143 & 0.447 & 0.107 & 0.750 \\
\hline
\end{tabular}

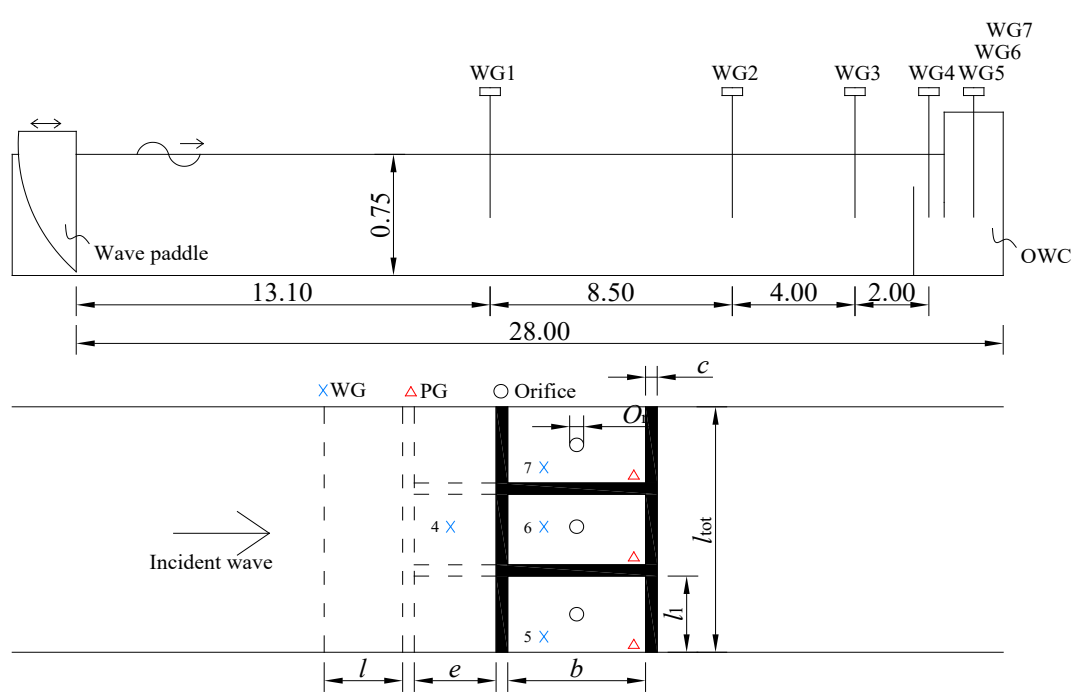

Figure 3 (a) Side view and (b) plan view of the U-OWC experimental model Vyzikas et al. [32].

The additional vertical duct influences greatly the hydrodynamic performance of the U-OWC device, thus it is necessary to re-verify the convergence of temporal and spatial discretization. Fig. 4 illustrates the time histories of the free-surface elevation at the chamber center and air pressure inside the chamber for three spatial steps of $\triangle x=$ $\lambda / 20, \lambda / 30$ and $\lambda / 40$ (where $\lambda$ is the wavelength) with $f=0.51 \mathrm{~Hz}$ and temporal step $\Delta t=$ $T / 60$. Similar tests are carried out for three temporal steps of $\triangle t=T / 40, T / 60$ and $T / 80$ as depicted in Fig. 5 , where $f=0.51 \mathrm{~Hz}$ and spatial step $\triangle x=\lambda / 30$. It should be noted that, due to the spatial step varying with the wavelength, meshes near the U-OWC device is refined to ensure the accuracy and stability of the simulation. From the figures,

it can be seen that the results with the intermediate spatial/temporal steps agree well 5 with those with the fine spatial/temporal steps. Finally, the mesh size $\Delta x$ and time step $6 \Delta t$ are chosen to be $\lambda / 30$ and $T / 60$, respectively. Additionally, the numerical stability of 7 the model is validated in Fig. 6. It can be seen that the two wave profiles agree well 
1 with each other both outside and inside the U-OWC chamber at $t=28 T$ and 30T. This

2 indicates that the present numerical model with inviscid assumption can reproduce the

3 interaction between waves and U-OWC device well. Concurrently, the damping zone

4 shows good wave absorbing ability at the left end of the flume. However, the wave

5 profile is irregular and higher-order harmonics occur due to the wave nonlinearity.

6

7

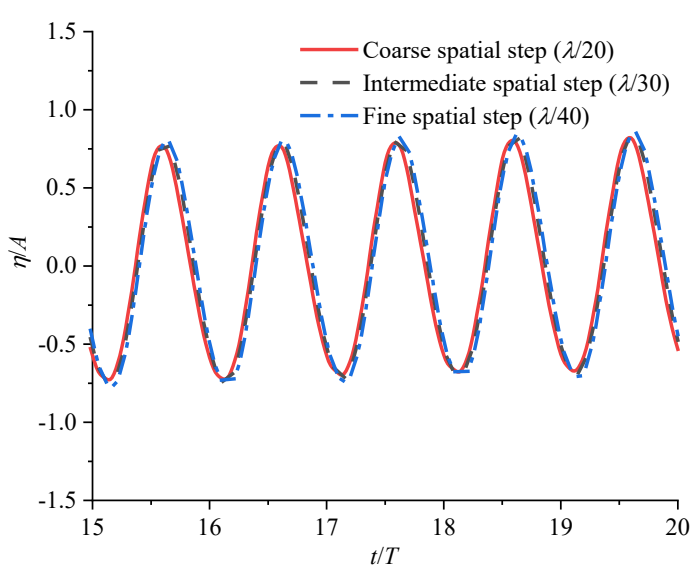

(a) Surface elevation at the chamber center

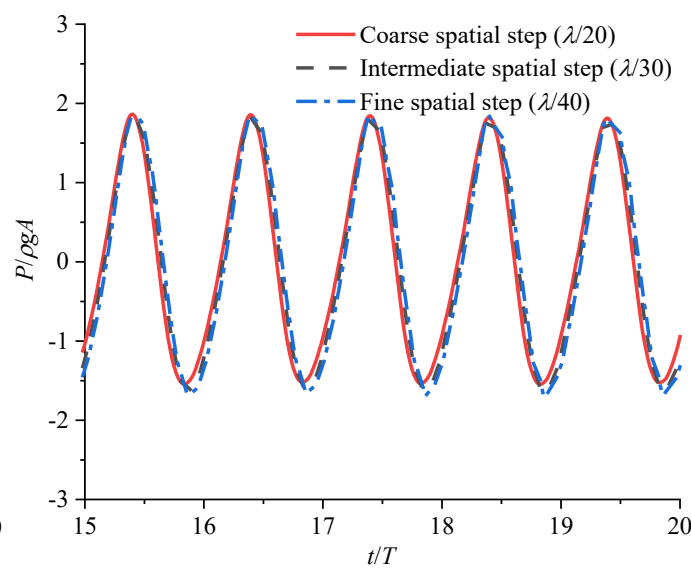

(b) Air pressure inside the chamber

Figure 4 Time series of surface elevation and air pressure for different spatial steps.

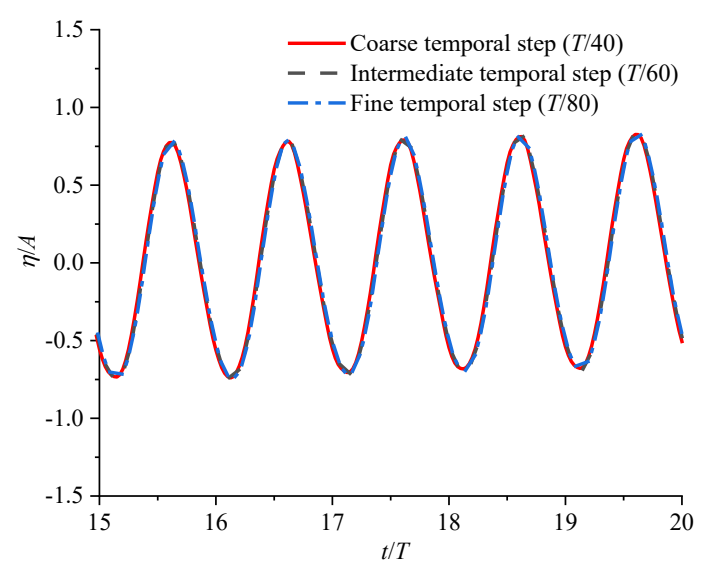

(a) Surface elevation at the chamber center

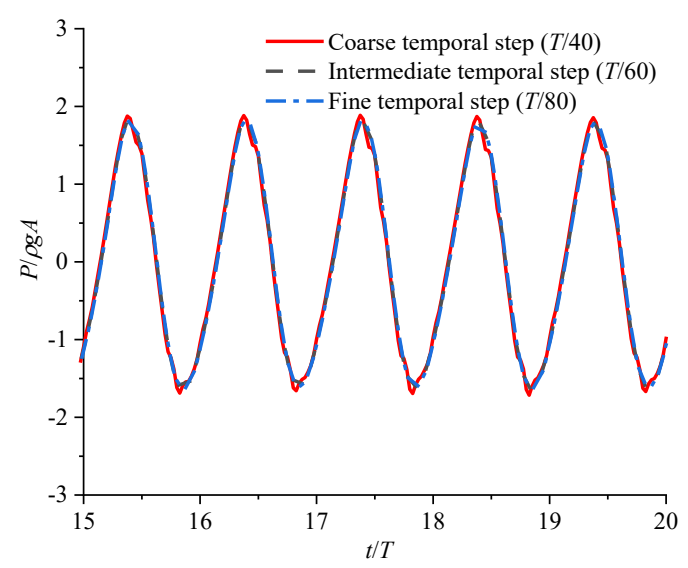

(b) Air pressure inside the chamber

Figure 5 Time series of surface elevation and air pressure for different temporal steps. 


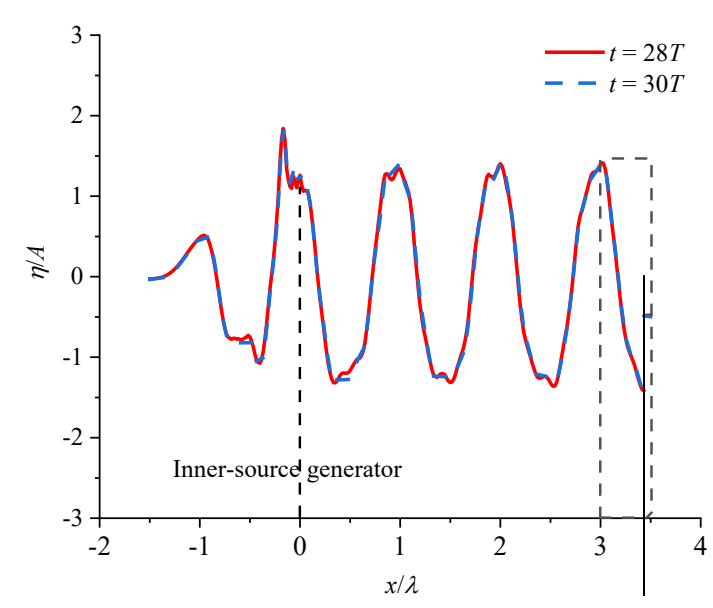

(a) Overall view

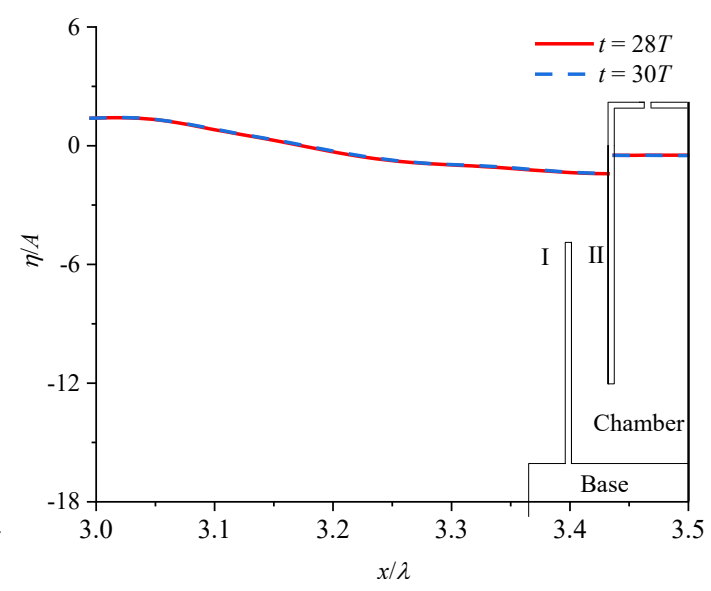

(b) Local detailed view

Figure 6 Snapshot of free-surface elevation along wave flume at $f=0.51 \mathrm{~Hz}$.

4

Time series of the surface elevation at the chamber center and air pressure in the chamber are depicted for different wave frequencies in Fig. 7 and Fig. 8, respectively.

The comparisons between the experiment [32] and the present numerical model are given in the figures. It can be observed that the surface elevation and air pressure predicted by the numerical model agree well with the experimental results. However, due to using linear pneumatic model, the high-order harmonics of the air pressure which occurs in the experimental data are not well predicted.

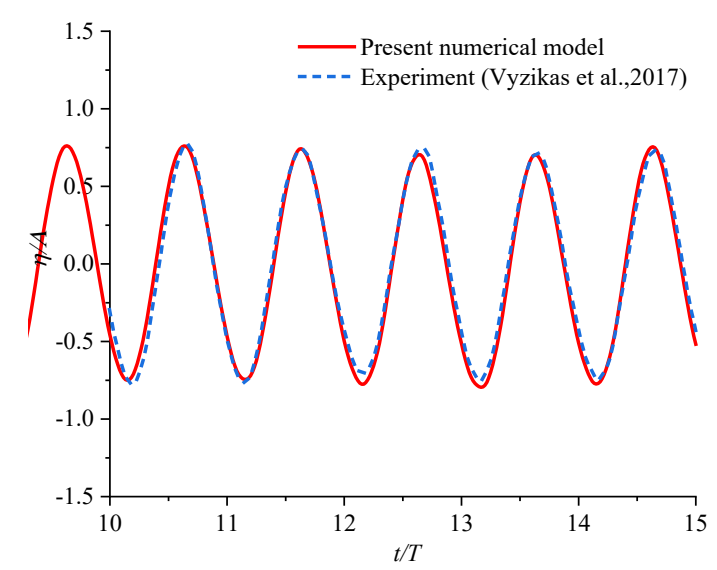

(a) $f=0.51 \mathrm{~Hz}$

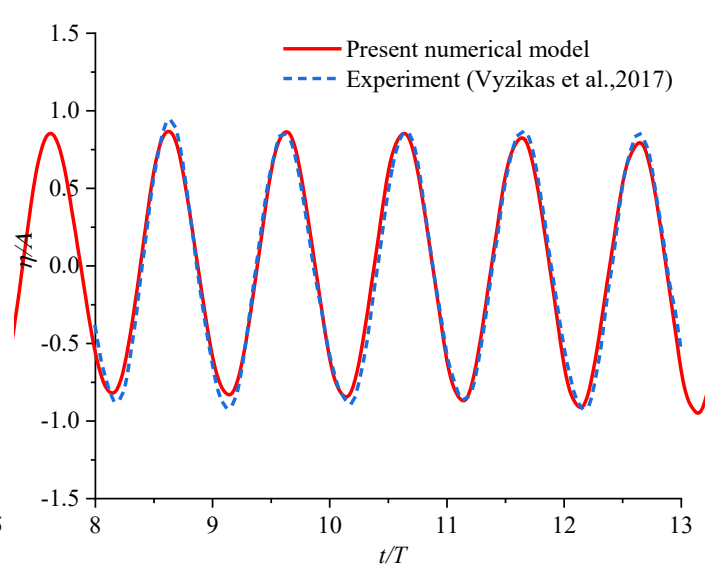

(b) $f=0.465 \mathrm{~Hz}$

Figure 7 Time series of surface elevation at the chamber center for different wave frequency. 


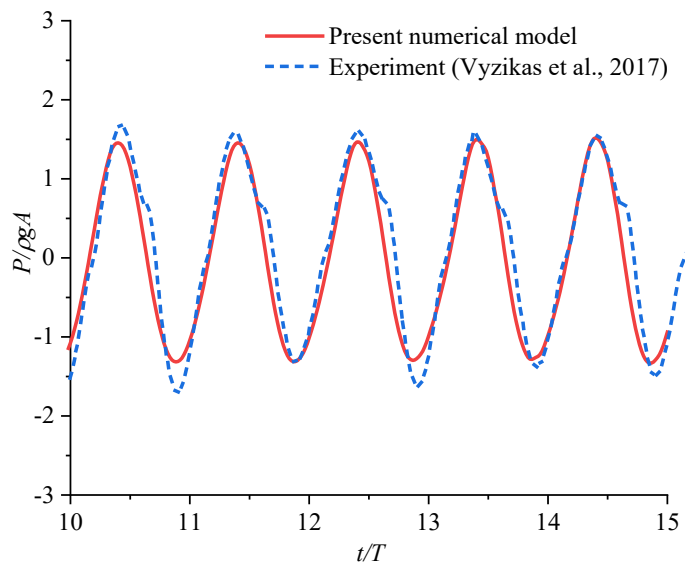

(a) $f=0.51 \mathrm{~Hz}$

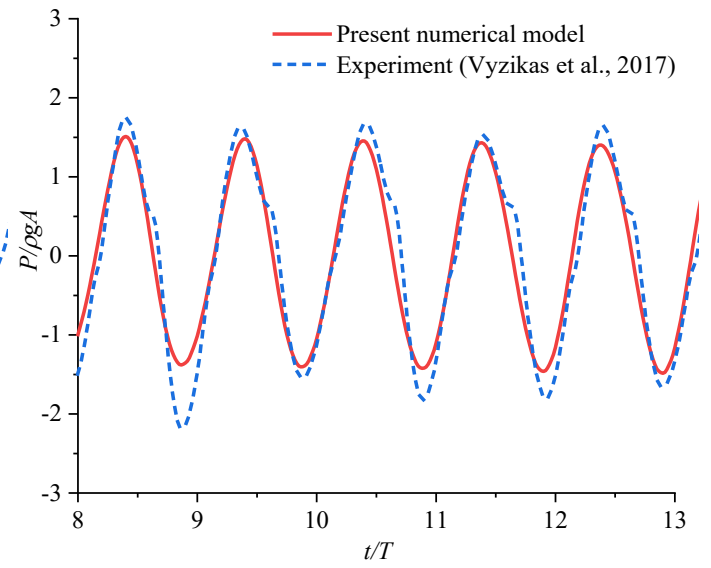

(b) $f=0.465 \mathrm{~Hz}$

Figure 8 Time series of air pressure in the chamber for different wave frequency.

Fig. 9 shows the variations of the surface elevation at the chamber center and simulated surface elevations and hydrodynamic efficiencies agree well with each other. As a whole, the numerical model can well simulate the main hydrodynamic behaviors 9 of the U-OWC device.

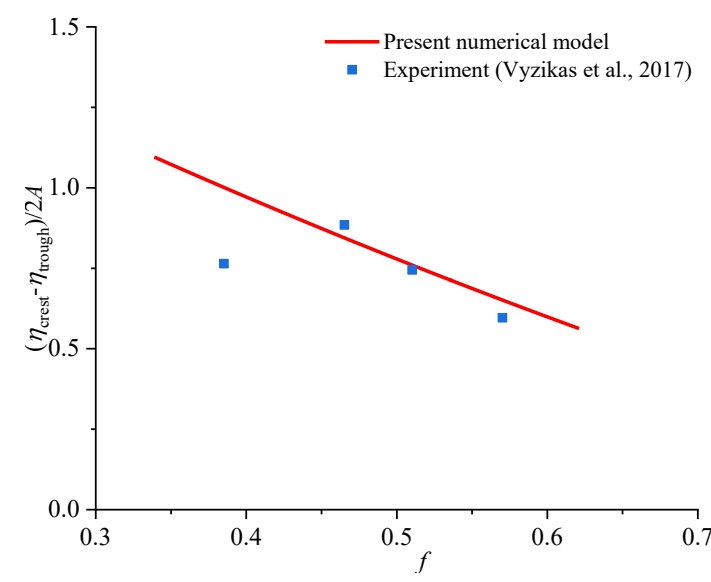

(a) Surface elevation

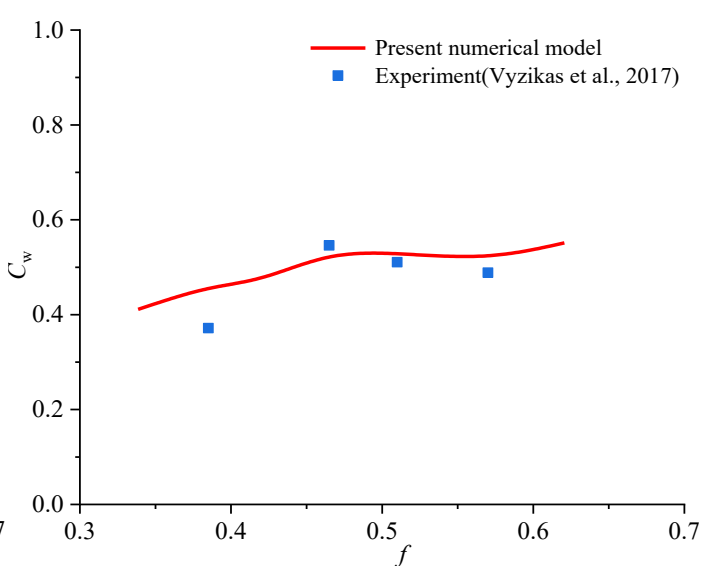

(b) Hydrodynamic efficiency

Figure 9 (a) Amplitude of surface elevation at the chamber center and (b) hydrodynamic efficiency versus wave frequency.

\section{Numerical results and discussions}


1 height, vertical duct width and thickness of barrier wall I) on the hydrodynamic

2 performance of the U-OWC device are performed. Totally, 78 cases are simulated. The

3 amplitudes of the free-surface elevation at the chamber center and the air pressure inside

4 the chamber are normalized as follows:

$$
\begin{gathered}
\eta_{\text {max }}=\frac{\left(\eta_{\text {crest }}-\eta_{\text {trough }}\right)}{2 A} \\
P_{\text {max }}=\frac{\left(P_{\text {crest }}-P_{\text {trough }}\right)}{2 \rho g A}
\end{gathered}
$$

where $\eta_{\max }$ and $P_{\max }$ denote the amplitudes of the free-surface elevation at the chamber center and the air pressure inside the chamber, respectively; $\eta_{\text {crest }}$ and $P_{\text {crest }}$ are the mean crest values of the time series of free-surface elevation and air pressure, respectively, $\eta_{\text {trough }}$ and $P_{\text {trough }}$ the corresponding mean trough values; $A$ is the incident wave amplitude and is taken as $0.04 \mathrm{~m}$.

\subsection{Effect of vertical duct height $w$}

To study the effect of the vertical duct height (which is equal to the height of the barrier wall I) on the hydrodynamic performance of the U-OWC device, the numerical simulation is carried out for different vertical duct heights $w$ with other geometrical parameters kept constant as listed in Tab. 1.

The variations of the surface-elevation amplitude at the chamber center and the air pressure in the chamber versus dimensionless wavenumber $k h$ for four vertical duct heights $w / h=0,0.297,0.596$ and 0.729 are shown in Fig. 10(a) and (b), respectively. Those symbols denote the original data and their fitting lines. It is found that the freesurface elevation at the chamber center and the air pressure inside the chamber increase with vertical duct height $w$ increasing. This is due to the fact that the distance between the upper opening and water surface decreases with the increase of the vertical-duct height, which leads to the increase of excitation pressure at the upper opening. On the other hand, according to the vertical eigenfunction in linear wave theory, the hydrodynamic pressure decays exponentially along the negative $z$ direction and its 
1 decay rate increases with wavenumber $k h$. This leads to the excitation pressure

2 difference between the tested cases increases with wavenumber $k h$. Eventually, the 3 effect of the vertical duct height on $P_{\max }$ increases with $k$ increasing in relatively low

4 frequency region (i.e., the difference between any two curves increases with $k h$ 5 increasing as shown in Fig. 10(b)). However, the difference decreases with the further 6 increase of $k h$ due to the increasing wave nonlinearity. In addition, an obvious peak is 7 observed from the air pressure curves at $w / h=0.729$. This may be due to the shallow 8 water effect for the quite small water depth above the Wall I at this case. Note that, due 9 to the limitation of the water depth, base thickness and incident wave conditions, and 10 the influence of the wave nonlinearity and reflection, the largest vertical duct height $w$ 11 is chosen to be $0.547 \mathrm{~m}$ (i.e., $w / h=0.729)$ in the present study.

12

13

14

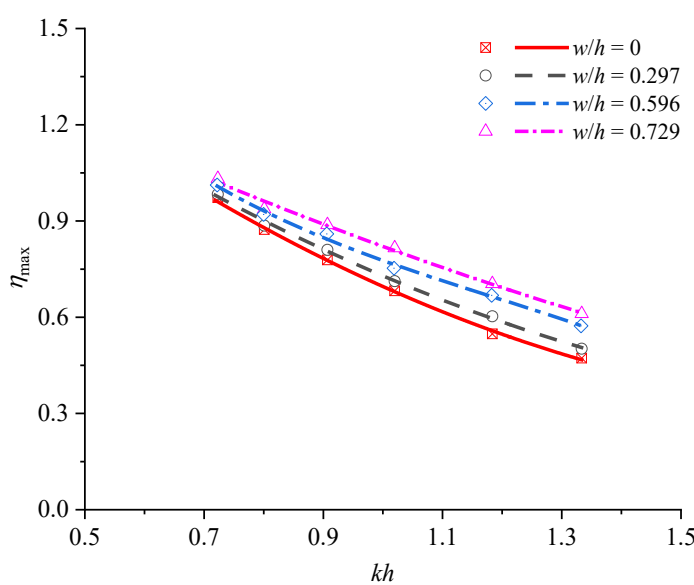

(a) Surface-elevation

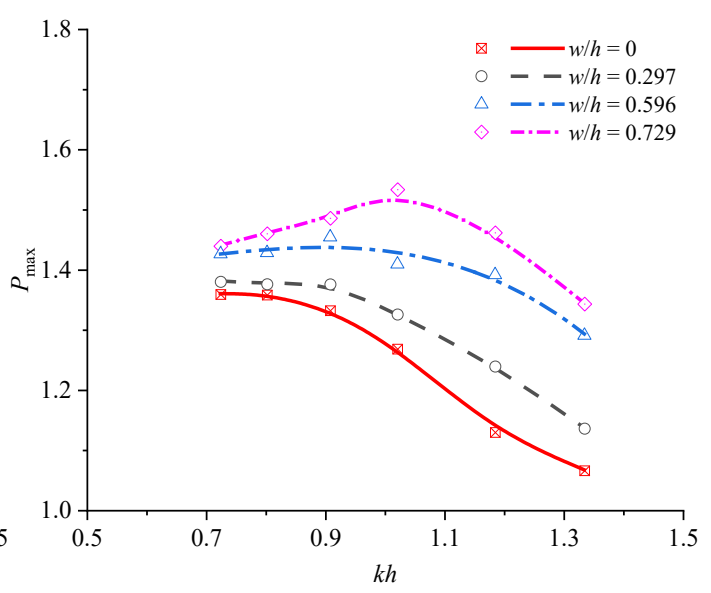

(b) Air pressure

Figure 10 Amplitudes of the (a) surface-elevation at chamber center and (b) air pressure inside the chamber versus dimensionless wavenumber $k h$ for different vertical duct heights.

As discussed above, the vertical duct height has significant influence on air pressure inside the chamber of the U-OWC device. To have a better understand of the influence of the vertical duct on the hydrodynamic performance, Fig. 11 shows the variation of hydrodynamic efficiency versus $k h$ for four vertical duct heights of $w / h=$ $0,0.297,0.596$ and 0.729 . The cubic fitting lines of the simulated scatter data are also 
1 plotted in the Fig. 11. The hydrodynamic efficiency $C_{\mathrm{w}}$ increases with the increase of

2 the vertical duct height $w$ and the effect of the vertical duct height on $C_{\mathrm{w}}$ increase as $k h$

3 increases in relatively low domain. According to Eq. (8), the hydrodynamic efficiency

$4 C_{\mathrm{w}}$ highly depends on the air pressure inside the chamber. Indeed, the similar

5 phenomenon also emerges on the air pressure inside the chamber as can be seen in Fig.

6 10(b). From Fig. 11, the optimal hydrodynamic efficiencies of $0.44,0.48,0.57$ and 0.64

$7 \quad$ occur at around $k h=0.86(w / h=0), k h=1.03(w / h=0.297), k h=1.18(w / h=0.596)$

8 and $k h=1.14(w / h=0.729)$, respectively. Additionally, one can easily draw a conclusion

9 from Figs. 10 and 11 that the U-OWC device performs better than the conventional

10 OWC device (i.e., the case of $w / h=0$ ) on the power absorption. The similar conclusion

11 was also obtained by Boccotti [22]. He pointed out that the better performance of the

$12 \mathrm{U}-\mathrm{OWC}$ device is due to the fact that the dynamic pressure exerted at the upper opening

13 of the U-OWC is larger than that at the opening of the conventional OWC device.

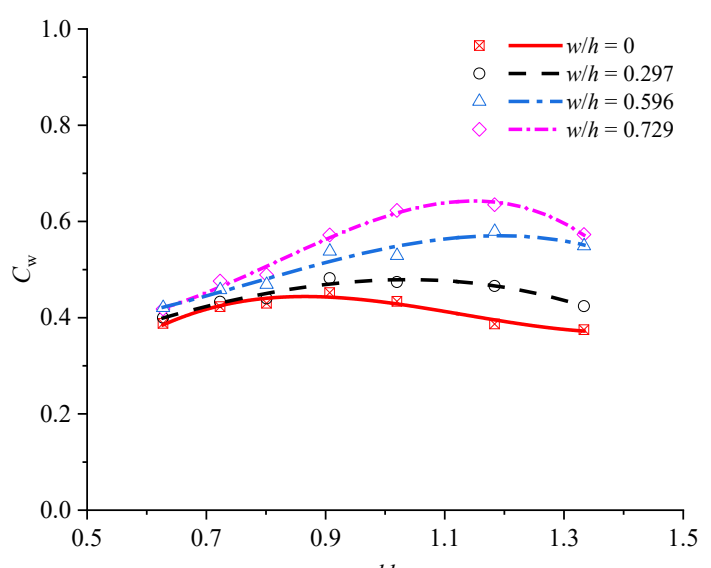

16 Figure 11 Hydrodynamic efficiency versus dimensionless wavenumber for different vertical duct heights.

Fig. 12 shows the free surface-elevation amplitude at the chamber center and the

21 air pressure inside the chamber versus $k h$ for five vertical duct widths of $e / h=0.067$, 
$10.191,0.381,0.572$ and 0.763 with remaining geometrical parameters kept constant as

2 listed in Tab. 1. As shown in Fig. 12(a), the free surface elevation $\eta_{\max }$ is insensitive to

3 the variation of vertical duct width and $\eta_{\max }$ decreases with the increase of $k h$

4 monotonously. Differently, the vertical duct width has a great influence on the air

5 pressure inside the chamber as shown in Fig. 12(b). Moreover, the surface elevation at

6 the chamber center and the air pressure inside the chamber increase with relative

7 vertical duct width $e / h$ in relatively low frequency region. However, in high frequency

8 region, $\eta_{\max }$ and $P_{\max }$ first increase and then decrease with the increase of $e / h$, and both

9 the increasing and decreasing rates increase with increasing $k h$.

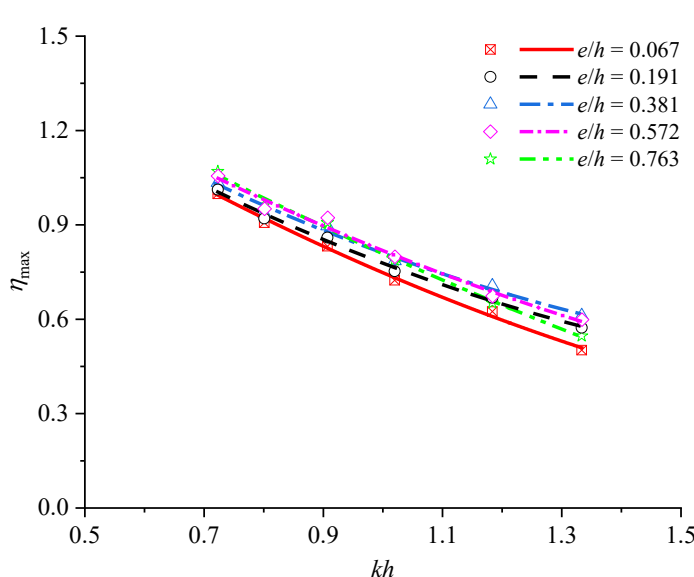

(a) Surface-elevation

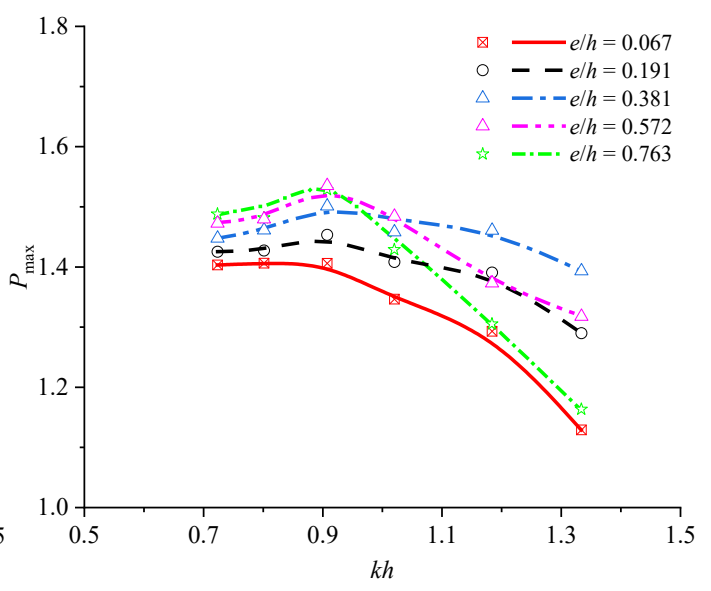

(b) Air pressure

Figure 12 Amplitudes of the (a) surface-elevation at the chamber center and (b) air pressure inside the chamber versus dimensionless wavenumber for different vertical duct widths.

Fig. 13 shows the variation of hydrodynamic efficiency versus $k h$ for various vertical duct widths. It can be seen that the hydrodynamic efficiency also displays similar variations to air pressure inside the chamber. A proper explanation for such phenomenon is that the hydrodynamic pressure at the upper opening changes little along the $x$ direction because the vertical duct width is small enough compared to the wavelength and wave nonlinearity is relatively small when $k h$ is relatively low. In view of this, the total hydrodynamic force exerted on the U-shaped water column almost 
1 increases linearly with the vertical duct width increasing, which strengthens the

2 oscillation of wave column and further leads to the increase of the air pressure inside

3 the chamber. However, the spatial inhomogeneity of the hydrodynamic pressure cannot

4 be ignored with the wavenumber increasing.

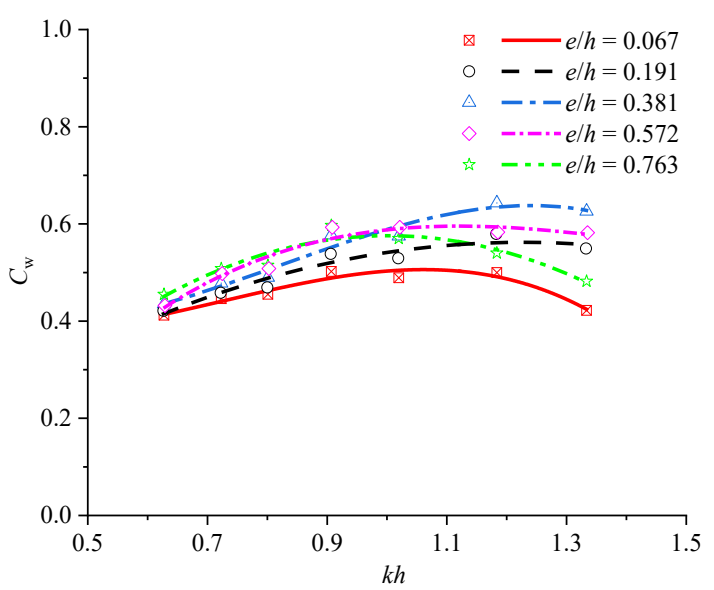

Figure 13 Hydrodynamic efficiency versus dimensionless wavenumber for different vertical duct widths.

Fig. 14(a) and (b) show the distribution of the free-surface elevation at the upper opening of the U-OWC device while wave crest arrives at barrier wall II for vertical duct width of $e / h=0.381$ and 0.763 , respectively. To make the horizontal position of the back wall to be 0 , a coordinate translation " $x-3.5 \lambda$ " is used in the Fig. 14 . The horizontal inhomogeneity can be clearly observed. It can be seen that the wave-surface slope before the device become steeper with the wave frequency $k h$ increasing from 0.627 to 1.184 due to the increase of wave nonlinearity. In other words, the free-surface elevation changes little along $x$ direction near the barrier wall II at $k h=0.627$; while it presents great variation along $x$ direction at $k h=1.184$, and this phenomenon becomes more obvious with increasing the vertical duct width. Such inhomogeneity of the surface elevation outside the chamber does not benefit the wave energy absorbing. 


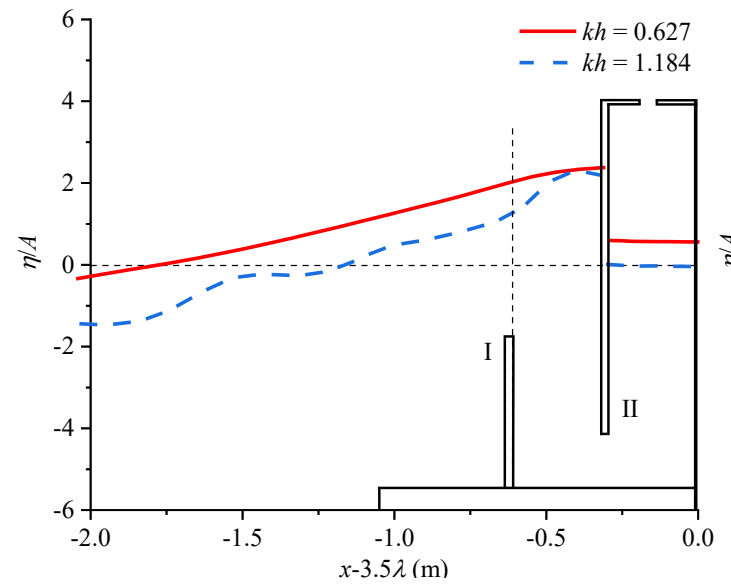

(a) $e / h=0.381$

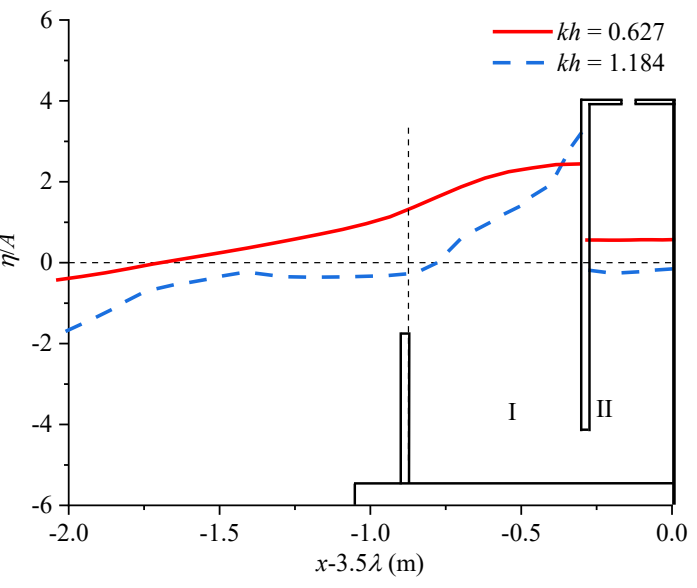

(b) $e / h=0.763$

Figure 14 Snapshot of free-surface elevation at the upper opening of the U-OWC device when wave crest arrives at barrier wall II for different wave frequencies.

\subsection{Effect of thickness of Wall I $c_{\mathrm{I}}$}

As shown in Fig. 2, $c_{\mathrm{I}}$ denotes the thickness of barrier wall I. By setting other parameters constant as listed in Tab. 1, the effect of four thicknesses of barrier wall I of $c_{\mathrm{I}} / h=0.032,0.048,0.064$ and 0.096 on the surface-elevation amplitude $\eta_{\max }$ at the chamber center and the air pressure $P_{\max }$ inside the chamber are shown in Fig. 15(a)

11 and (b), respectively. It can be seen that $\eta_{\max }$ and $P_{\max }$ increase with the thickness of

12 barrier wall I increasing, but the growing trend weakens obviously with the increase of thickness. Besides, the barrier wall I thickness has little effect on the shape of the curves of the surface elevation at the chamber center and the air pressure inside the chamber.

15 For all tested cases, $\eta_{\max }$ decreases with the increase of $k h$, and $P_{\max }$ increases initially 16 to its peak and then decreases with $k h$ increasing. Fig. 16 shows the effect of the barrier wall I thickness on the hydrodynamic efficiency. It can be observed that the larger thickness of barrier wall I, the higher hydrodynamic efficiency and lower resonant

19 frequency. The optimal hydrodynamic efficiencies for $c_{\mathrm{I}} / h=0.032,0.0480 .064$ and 200.096 occur at around $k h=1.20,1.17,1.14$ and 1.10 with its hydrodynamic efficiency $210.56,0.67,0.68$ and 0.71 , respectively. Additionally, similar to the trend of the surface 
1 elevation and air pressure inside the chamber, the hydrodynamic efficiency also

2 increases with the thickness of barrier wall I in a declining rate. It is worth noting that

3 the construction cost increases linearly with the increase of the thickness of barrier wall

4 I. Thus, from the engineering point of view, it needs to balance the construction cost 5 and the hydrodynamic performance of the U-OWC device.

6

7

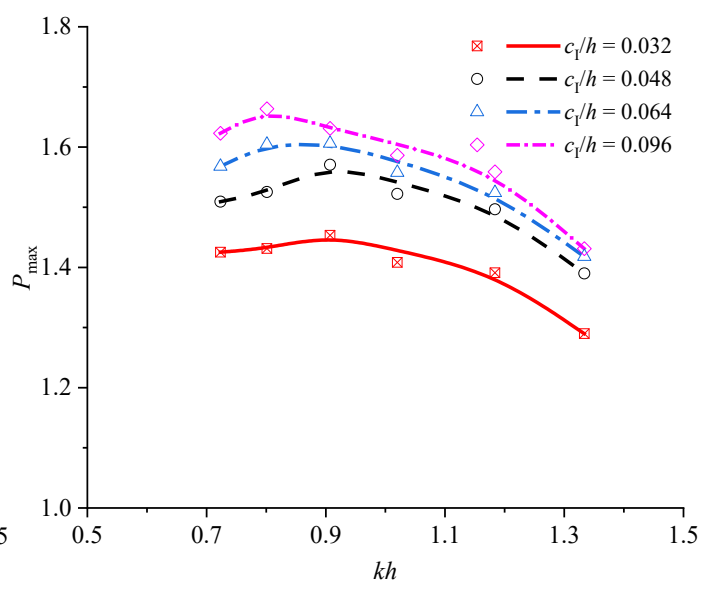

(b) Air pressure

Figure 15 Amplitudes of the (a) surface-elevation at the chamber center and (b) air pressure inside the chamber versus dimensionless wavenumber for different thicknesses of wall I.

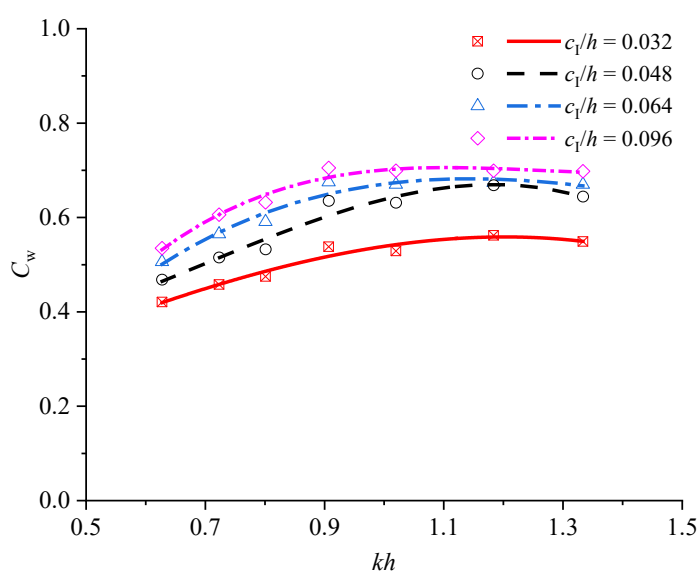

Figure 16 Hydrodynamic efficiency versus dimensionless wavenumber for different thicknesses of wall I. 


\section{Conclusions}

In the present investigation, the hydrodynamic performance of the U-OWC Wave Energy Converter is analyzed. Based on the time-domain higher-order boundary element method (HOBEM), a 2-D fully nonlinear numerical wave flume with a UOWC device is implemented to study the effects of the geometric parameters on the hydrodynamic efficiency, free-surface elevation and air pressure inside the U-OWC chamber. In the numerical simulation, the inner-source technique is used to generate incident wave to avoid re-reflection, the mixed Eulerian-Lagrangian technique and the $4^{\text {th }}$-order Runge-Kuatta method are applied as the time marching technology. The linear relationship between the air pressure inside the chamber and the air velocity at the orifice is assumed to establish the pneumatic model.

The numerical results indicate that the predicted surface elevation at the chamber center and the air pressure inside the chamber agree well with the published experimental data, generally. Further, the effects of the vertical duct height, vertical duct width and thickness of barrier wall I on hydrodynamic performance of the U-OWC device are investigated. The increase of vertical duct height can improve the hydrodynamic efficiency because the upper opening of vertical duct is closer to free surface. Therefore, the U-OWC device performs better than the conventional OWC device. As for the vertical duct width, it is observed that a larger vertical duct width leads to a larger hydrodynamic efficiency in relatively low frequency domain. However, $\eta_{\max }$ and $P_{\max }$ initially increase and then decrease with the increase of vertical duct width in the high frequency region. This is due to the fact that the horizontal spatial inhomogeneity of the excitation pressure at the upper opening is amplified with the decrease of wavelength (increasing wave frequency). The hydrodynamic efficiency is found to increase with the increase of thickness of barrier wall I in a declining rate. However, the construction cost increases linearly with the increase of the thickness of barrier wall I as well. Thus, from the engineering point of view, a comprehensive 
1 consideration of the construction cost and hydrodynamic performance is necessary in

2 the design and construction of a U-OWC device.

\section{Acknowledgments}

[1] A. G. L. Borthwick. Marine Renewable Energy Seascape. Engineering. 2016;2:69-78.

[2] D. V. Evans, R. Porter. Hydrodynamic characteristics of an oscillating water column device. Appl Ocean Res. 1995;15:155-64.

[3] H. Martins-rivas, C. C. Mei. Wave power extraction from an oscillating water column along a straight coast. Ocean Engineering. 2009;36:426-33.

[4] S. Zheng, A. Antonini, Y. Zhang, D. Greaves, J. Miles, G. Iglesias. Wave power extraction from multiple oscillating water columns along a straight coast. Journal of Fluid Mechanics. 2019;878:445-80.

[5] Z. Deng, L. Wang, X. Zhao, P. Wang. Wave power extraction by a nearshore oscillating water column converter with a surging lip-wall. Renewable Energy. 2020;146:662-74.

[6] D. Z. Ning, R. Q. Wang, Q. P. Zou, B. Teng. An experimental investigation of hydrodynamics of a fixed OWC Wave Energy Converter. Applied Energy. 2016;168:636-48.

[7] R.-q. Wang, D.-z. Ning. Dynamic analysis of wave action on an OWC wave energy converter under the influence of viscosity. Renewable Energy, 2020, https://doi.org/10.1016/j.renene.2020.01.007.

[8] A. F. O. Falcão, J. C. C. Henriques, J. J. Cândido. Dynamics and optimization of the OWC spar buoy wave energy converter. Renewable Energy. 2012;48:369-81.

[9] R. P. F. Gomes, J. C. C. Henriques, L. M. C. Gato, A. F. O. Falcão. Wave power extraction of a heaving floating oscillating water column in a wave channel. Renewable Energy. 2016;99:1262-75.

[10] A. J. C. Crespo, C. Altomare, J. M. Domínguez, J. González-Cao, M. Gómez- 
Gesteira. Towards simulating floating offshore oscillating water column converters with Smoothed Particle Hydrodynamics. Coastal Engineering. 2017;126:11-26.

[11] F. He, Z. Huang. Hydrodynamic performance of pile-supported OWC-type structures as breakwaters: An experimental study. Ocean Engineering. 2014;88:618-26.

[12] K. Rezanejad, J. Bhattacharjee, C. Guedes Soares. Analytical and numerical study of dual-chamber oscillating water columns on stepped bottom. Renewable Energy. 2015;75:272-82.

[13] F. He, J. Leng, X. Zhao. An experimental investigation into the wave power extraction of a floating box-type breakwater with dual pneumatic chambers. Applied Ocean Research. 2017;67:21-30.

[14] D.-z. Ning, R.-q. Wang, L.-f. Chen, K. Sun. Experimental investigation of a landbased dual-chamber OWC wave energy converter. Renewable and Sustainable Energy Reviews. 2019;105:48-60.

[15] D.-z. Ning, Y. Zhou, R. Mayon, L. Johanning. Experimental investigation on the hydrodynamic performance of a cylindrical dual-chamber Oscillating Water Column device. Applied Energy. 2020;260.

[16] F. Mahnamfar, A. Altunkaynak. Comparison of numerical and experimental analyses for optimizing the geometry of OWC systems. Ocean Engineering. 2017;130:10-24.

[17] N. Dizadji, S. E. Sajadian. Modeling and optimization of the chamber of OWC system. Energy. 2011;36:2360-6.

[18] M. Kharati-Koopaee, M. Fathi-Kelestani. Assessment of oscillating water column performance: Influence of wave steepness at various chamber lengths and bottom slopes. Renewable Energy. 2020:1595-608.

[19] A. F. O. Falcão. The shoreline OWC wave power plant at the Azores,. Proceedings of the Fourth European Wave Energy Conference. Aalborg, Denmark,2000.

[20] A. F. O. Falcão, J. C. C. Henriques. Oscillating-water-column wave energy converters and air turbines: A review. Renewable Energy. 2016;85:1391-424.

[21] M. Suzuki, C. Arakawa, S. Takahashi. Performance of wave power generating system installed in breakwater at Sakata port in Japan. Proceedings of the Fourteenth International Offshore and Polar Engineering Conference. Toulon, France,May 23-28, 2004.

[22] P. Boccotti. Comparison between a U-OWC and a conventional OWC. Ocean Engineering. 2007;34:799-805.

[23] P. Boccotti. On a new wave energy absorber. Ocean Engineering. 2003;30:1191200.

[24] P. Boccotti. Caisson breakwaters embodying an OWC with a small openingPart I: Theory. Ocean Engineering. 2007;34:806-19.

[25] P. Boccotti, P. Filianoti, V. Fiamma, F. Arena. Caisson breakwaters embodying 
an OWC with a small opening-Part II: A small-scale field experiment. Ocean

[26] G. Malara, F. Arena. Analytical modelling of an U-Oscillating Water Column and performance in random waves. Renewable Energy. 2013;60:116-26.

[27] G. Malara, A. Romolo, V. Fiamma, F. Arena. On the modelling of water column oscillations in U-OWC energy harvesters. Renewable Energy. 2017;101:964-72.

[28] G. Malara, R. P. F. Gomes, F. Arena, J. C. C. Henriques, L. M. C. Gato, A. F. O. Falcão. The influence of three-dimensional effects on the performance of U-type oscillating water column wave energy harvesters. Renewable Energy. 2017;111:506-22.

[29] P. D. Spanos, F. M. Strati, G. Malara, F. Arena. An approach for non-linear stochastic analysis of U-shaped OWC wave energy converters. Probabilistic Engineering Mechanics. 2018;54:44-52.

[30] G. Malara, F. Arena. Response of U-Oscillating Water Column arrays: semianalytical approach and numerical results. Renewable Energy. 2019;138:115265.

[31] F. M. Strati, G. Malara, V. Laface, A. Romolo. A Control Strategy for PTO Systems in a U-OWC Device. Proceedings of the ASME 2015 34th International Conference. Newfoundland, Canada2015.

[32] T. Vyzikas, S. Deshoulières, M. Barton, O. Giroux, D. Greaves, D. Simmonds. Experimental investigation of different geometries of fixed oscillating water column devices. Renewable Energy. 2017;104:248-58.

[33] F. Arena, L. Daniele, V. Fiamma, M. Fontana, G. Malara, G. Moretti, et al. Field Experiments on Dielectric Elastomer Generators Integrated on a U-OWC Wave Energy Converter. Volume 10: Ocean Renewable Energy2018.

[34] P. Boccotti. Design of breakwater for conversion of wave energy into electrical energy. Ocean Engineering. 2012;51:106-18.

[35] F. Arena, A. Romolo, G. Malara. On Design and Building of a U-OWC Wave Energy Converter in the Mediterranean Sea: A Case Study. Proceedings of the ASME 2013 32nd International Conference. Nantes, France2013.

[36] D. Z. Ning, J. Shi, Q. P. Zou, B. Teng. Investigation of hydrodynamic performance of an OWC (oscillating water column) wave energy device using a fully nonlinear HOBEM (higher-order boundary element method). Energy. 2015;83:177-88.

[37] R. Q. Wang, D. Z. Ning, C. W. Zhang, Q. P. Zou, Z. Liu. Nonlinear and viscous effects on the hydrodynamic performance of a fixed OWC wave energy converter. Coastal Engineering. 2018;131:42-50.

[38] W. Koo, M. H. Kim, D. H. Lee. Nonlinear Time-domain Simulation of Pneumatic Floating Breakwater. International Journal of Offshore and Polar Engineering. 2006;16:25-32. 